\title{
Enhancement of DNA vaccine potency against herpes simplex virus 1 by co-administration of an interleukin-18 expression plasmid as a genetic adjuvant
}

\author{
Mingzhao Zhu, ${ }^{1}$ Xuemei Xu, ${ }^{1}$ Hongwei Liu, ${ }^{2}$ Xiaojuan Liu, ${ }^{1}$ Sheng Wang, ${ }^{3}$ \\ Fangtian Dong, ${ }^{3}$ Baoling Yang ${ }^{2}$ and Guoxing Song ${ }^{1}$
}

Correspondence

Guoxing Song

songgx@ms.imicams.ac.cn

Received 13 June 2002

Accepted 15 November 2002
${ }^{1,3}$ Department of Biophysics, Institute of Basic Medical Sciences ${ }^{1}$ and Department of Ophthalmology, Peking Union Hospital ${ }^{3}$, Chinese Academy of Medical Sciences \& Peking Union Medical College, Beijing 100005, PR China

${ }^{2}$ Department of Pharmacology, Beijing Institute of Ophthalmology, Beijing 100062, PR China

In this study, the immune-modulatory and vaccine effects of using an interleukin (IL)-18 expression plasmid as a genetic adjuvant to enhance DNA vaccine-induced immune responses were investigated in a mouse herpes simplex virus 1 (HSV-1) challenge model. BALB/c mice were immunized by three intramuscular inoculations of HSV-1 glycoprotein D (gD) DNA vaccine alone or in combination with a plasmid expressing mature IL-18 peptide. Both the serum IgG2a/lgG1 ratio and Thelper 1-type (Th1) cytokines [IL-2 and interferon (IFN)- $\gamma$ ] were increased significantly by the co-injection of the IL-18 plasmid compared with the injection of gD DNA alone. However, the production of IL-10 was inhibited by IL-18 plasmid co-injection. Furthermore, IL-18 plasmid coinjection efficiently enhanced antigen-specific lymphocyte proliferation and the delayed-type hypersensitivity response. When mice were challenged with HSV-1 at the cornea, co-injection of IL18 plasmid with gD DNA vaccine showed significantly better protection, manifested as lower corneal lesion scores and faster recovery. These experiments indicate that co-injection of an IL-18 plasmid with gD DNA vaccine efficiently induces Th1-dominant immune responses and improves the protective effect against HSV-1 infection.

\section{INTRODUCTION}

Nucleic acid immunization is an important vaccination strategy that has many characters desirable for an ideal vaccine, including induction of broad immune responses (humoral and cellular), long-lasting immunity and simple and cheap production. This technique is being explored as a vaccination strategy against a variety of infectious diseases, autoimmune diseases and cancers. The first generation of DNA-immunization experiments have shown that delivery of DNA constructs encoding a specific immunogen into the host could elicit effective immune responses in vivo in a safe and well-tolerated manner in various model systems. However, more efficacious and specific immune responses against the target pathogen are required in order to enhance its clinical utility. One strategy is the use of molecular adjuvants. Molecular or genetic adjuvants are different from the traditional adjuvants in that they consist of gene-expression constructs encoding immunologically important molecules,

Abbreviations: DTH, delayed-type hypersensitivity; gD, glycoprotein D; HSV-1, herpes simplex virus 1 ; IFN, interferon; IL, interleukin. such as cytokines, chemokines and co-stimulatory molecules (Iwasaki et al., 1997; Kim et al., 1997, 2000; Sin et al., 1999). Previous reports have shown that co-administration of genetic adjuvant constructs with immunogen constructs can modulate antigen-specific immune responses (Kim et al., 1998, 1999a).

Interleukin (IL)-18, first designed as an interferon (IFN)- $\gamma$ inducing factor, is a recently identified cytokine of the T helper 1 (Th1) type. It has been known to induce IFN- $\gamma$ production by both $\mathrm{CD} 4^{+} \mathrm{T}$ cells and natural killer $(\mathrm{NK})$ cells (Okamura et al., 1995; Ushio et al., 1996) and to stimulate naive $\mathrm{T}$ cells to promote the development of Th1 cells (Kohno et al., 1997). Since IFN- $\gamma$ is one of the most important cytokines that contributes to host defence, a cytokine capable of up-regulating IFN- $\gamma$ should also play a key role in host defence. Indeed, IL-18 plays a critical role in the eradication of various pathogens including Leishmania major (Ohkusu et al., 2000), Mycobacterium leprae (Garcia et al., 1999), encephalomyocarditis virus (Tovey et al., 1999), human immunodeficiency virus (Billaut-Mulot et al., 2001) and herpes simplex virus (HSV) (Fujioka et al., 1999). 
HSV is the causative agent of a spectrum of human diseases including ocular infections, encephalitis and genital infection. Immunizing animals with recombinant glycoprotein D (gD) protein, one of 11 known HSV glycoproteins, provides effective protection against both HSV-1 and HSV-2 infection in mice (Keadle et al., 1997; Corey et al., 1999). Similarly, gD DNA vaccine also protects mice against challenge by HSV-1 or HSV-2 (Bourne et al., 1996; Inoue et al., 2000). Since it has the best immunogenicity among the 11 glycoproteins and is highly conserved and antigenically cross-reactive between HSV-1 and HSV-2, gD has become the most important candidate immunogen.

Several cytokine genetic adjuvants including IL-2, IL-12 and granulocyte-macrophage colony-stimulating factor have been used in combination with gD DNA vaccine in immunization protocols to induce more protective immune responses against HSV (Sin et al., 1998, 1999; Inoue et al., 2000). The use of a plasmid encoding the Th1-inducing cytokine IL-18 has also been reported to exert immunomodulatory properties in vivo (Kim et al., 1999b; Kremer et al., 1999). Based on these observations, in this study, we tested the immune-modulatory and vaccine effects of using an IL-18 expression plasmid as a genetic adjuvant to enhance gD DNA vaccine-induced preventive immune responses in a mouse HSV-1 challenge model.

\section{METHODS}

Virus. HSV-1 KOS strain was propagated in 2BS cells. At maximum cytopathic effect, the viruses were harvested by three cycles of freezing and thawing. After centrifugation at 5000 r.p.m. for $5 \mathrm{~min}$, the supernatant was aliquotted and stored at $-80^{\circ} \mathrm{C}$ before use.

DNA plasmids. The two plasmids pgD (pcDNA3.1-gD), encoding HSV-1 gD protein, and pIL-18 (pcDNA3.1-IL-18), the IL-18 expression plasmid, were constructed and identified in our laboratory as described previously (Zhu et al., 2002). Briefly, the whole gD gene was amplified by PCR from the HSV-1 genome and then inserted into pcDNA3.1 to give the $\mathrm{gD}$ expression plasmid. pIL-18 was constructed by inserting the kappa leader sequence-fused mature human IL-18 cDNA, which was obtained by PCR from a human tonsil cDNA library, into the pcDNA3.1 backbone. For DNA immunization, plasmid DNA of pcDNA3.1, pgD and PIL-18 was prepared using the Endofree Plasmid Giga kit (Qiagen).

DNA inoculation of mice. Six- to eight-week-old female BALB/c mice were used in this study. Two days before DNA inoculation, the quadriceps muscles were injected with $100 \mu \mathrm{l}$ of a solution containing $0 \cdot 25 \%$ bupivacaine hydrochloride to enhance subsequent DNA absorption. For DNA inoculation, $100 \mu \mathrm{g}$ of each DNA construct in PBS was injected into the same region of the muscle as the bupivacaine injection. Since our preliminary experiments showed no difference in immune responses when inoculating $100 \mu \mathrm{g} \mathrm{pgD}$ either alone or together with $100 \mu \mathrm{g}$ empty pcDNA3.1, in our present study, the gD-immunization group was injected with pgD plasmid alone. Co-administration of pIL18 involved mixing the chosen plasmids prior to injection. The mice were boosted with the same dose at weeks 2 and 4 .

ELISA. The induced antibody response was quantified by ELISA as described previously (Zhu et al., 2002). In particular, HSV-1 gD (ViroStat) at $2 \mu \mathrm{g} \mathrm{ml}^{-1}$ in carbonate/bicarbonate buffer was used as a coating antigen. To determine ELISA titres, sera were serially diluted to
$1: 1000,1: 2000,1: 3000,1: 4000,1: 5000,1: 6000,1: 7000$ and $1: 8000$. The end-point titres were expressed as the last reciprocal serum dilution that gave an absorbance greater than $0 \cdot 1$ and at least twofold higher than the absorbance of control mouse sera at the same dilution. For the determination of relative levels of $\mathrm{gD}$-specific IgG subclasses, an ELISA was performed with biotinylated goat anti-mouse IgG1 and IgG2a (Pharmingen) and horseradish peroxidase-conjugated streptavidin.

Splenocyte-proliferation assay. Lymphocytes were prepared from mouse spleens. The isolated cell suspensions were resuspended to a

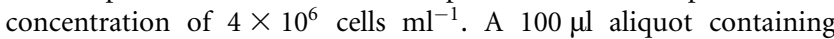
$4 \times 10^{5}$ cells was added immediately to each well of a 96-well flatbottomed microtitre plate. One-hundred microlitres of HSV-1 gD protein was added to wells in duplicate, to a final concentration of $10 \mu \mathrm{g}$ $\mathrm{ml}^{-1}$. The cells were incubated at $37^{\circ} \mathrm{C}$ in $5 \% \mathrm{CO}_{2}$ for $66 \mathrm{~h}$. One microcurie of ${ }^{3} \mathrm{H}$-thymidine was added to each well and the cells were incubated continuously for $6-8 \mathrm{~h}$ at $37^{\circ} \mathrm{C}$. The plate was harvested and the amount of ${ }^{3} \mathrm{H}$-thymidine incorporated was measured in a Beta

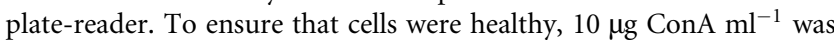
used as a polyclonal stimulator-positive control. The stimulation index (SI) was calculated as experimental c.p.m./spontaneous c.p.m.

Th1 and Th2 cytokine assays. Cytokines were assayed in supernatants of spleen cells $\left(4 \times 10^{6}\right.$ cells ml $\left.^{-1}\right)$ cultured for $72 \mathrm{~h}$ in the presence of HSV-1 gD protein $\left(10 \mu \mathrm{g} \mathrm{ml}^{-1}\right)$. IL-2, IL-10 and IFN- $\gamma$ levels were determined by using commercial cytokine kits (Diaclone).

Delayed-type hypersensitivity (DTH) assay. Two weeks after the final immunization, mice were injected in the dorsal side of each pinna. The right pinna was injected with $10 \mu \mathrm{l}\left(1 \times 10^{6}\right.$ p.f.u. $\left.\mathrm{ml}^{-1}\right) \mathrm{UV}$ lightinactivated HSV antigen. The left pinna was injected with the same amount of supernatant of 2BS cell lysate as a control. Forty-eight $h$ later, the thickness of each ear was measured with an engineer's micrometer. The DTH response in each mouse was expressed as the difference in thickness between the left and right pinnae.

Virus challenge of the cornea and evaluation of the results. Two weeks after the last immunization, all corneas of the mice were scarified in a criss-cross pattern 10 times with a 27 -gauge needle. Five microlitres of solution containing $1 \times 10^{6}$ p.f.u. virus $\mathrm{ml}^{-1}$ was instilled into the conjunctival sac of each eye.

Clinical evaluation of viral infection challenge was carried out as described previously (Inoue et al., 2000). Briefly, every day from day 1 to day 5 and on days 7 and 9 after the viral challenge, the same observer examined the eyes with a slit-lamp biomicroscope and scored the severity of epithelial lesions by the following criteria: 0 , no epithelial lesion or punctate epithelial erosion; 1, stellate keratitis or residue of dendritic keratitis; 2 , dendritic keratitis occupying less than a quarter of the cornea; 3, dendritic keratitis occupying a quarter to half of the cornea; 4, dendritic keratitis extending over more than half of the cornea.

Statistical analysis. Statistical analysis was performed using Student's $t$ test and the Kruskal-Wallis $\mathrm{H}$ test. Values were compared between different immunization groups. $P$ values $<0.05$ were considered statistically significant.

\section{RESULTS}

\section{Antibody responses}

To determine whether co-injection of IL-18 expression plasmid (pIL-18) with gD DNA vaccine ( $\mathrm{pgD}$ ) could influence the humoral immune response against $\mathrm{gD}$, sera obtained 2 weeks after the final DNA inoculation were tested 
by ELISA. When pIL-18 was co-injected, the geometric mean titre was increased to about 6000 , significantly higher than the group immunized with $\mathrm{pgD}$ alone, in which group the mean titre was only about $4400(P<0 \cdot 05)$.

To characterize the immune response elicited against $\mathrm{gD}$ antigen, IgG isotypes were identified. The results showed that pIL-18 co-injection resulted in a significant increase in the IgG2a/IgG1 ratio, indicating the dominance of Th1 cell function in the humoral immune response (Table 1).

\section{Splenocyte proliferation}

Splenocyte proliferation is a standard parameter used to evaluate the potency of cell-mediated immunity. It was carried out 2 weeks after the last immunization. As shown in Table 2, a low background level of proliferation was observed in the negative control. However, gD DNA vaccinestimulated cells had an enhanced proliferative response. When the mice were co-injected with pIL-18, the level of splenocyte proliferation was further increased.

\section{Levels of Th1 and Th2 cytokines}

Th1 and Th 2 cytokines play different roles in the polarization of immune responses. Th1 cytokines are thought to drive induction of cellular immunity, whereas Th2 cytokines preferentially drive humoral immunity. To understand the role of IL-18 in the development of immune responses, we examined the effect of co-injection of pgD with and without pIL-18 on changes in Th1 and Th2 phenotypes. As shown in Fig. 1, co-injection of pIL-18 significantly increased the production of IFN- $\gamma$ and IL-2 (Th1-type) compared with pgD alone. In contrast, the IL-10 (Th2-type) level was decreased by pIL-18 co-injection.

\section{DTH}

As shown in Table 3, intradermal injection of HSV antigen in the pinna resulted in a significant DTH response in mice immunized with $\mathrm{pgD}$ compared with negative controls. The DTH response was further increased in the pgD+pIL-18 group to a level significantly higher than that in the group that received $\mathrm{pgD}$ alone $(P<0 \cdot 05)$.
Table 2. Splenocyte proliferation levels after in vitro stimulation with $\mathrm{gD}$ protein

Groups of mice $(n=2)$ were immunized with $100 \mu \mathrm{g} p g D$ alone or with $100 \mu \mathrm{g}$ pIL-18 at 0,2 and 4 weeks; pcDNA3.1-immunized mice were used as a negative control. Two weeks after the final immunization, mice were sacrificed and splenocytes were isolated. Splenocytes were then stimulated with $10 \mu \mathrm{ggD}$ protein $\mathrm{ml}^{-1}$ or $10 \mu \mathrm{g}$ ConA ml ${ }^{-1}$ as a positive control. After 3 days of stimulation, the cells were harvested and incorporated ${ }^{3} \mathrm{H}$ was counted. The experiment was repeated with similar results; data from the two experiments were included in the analysis. The ConA control group showed an SI of 6.1. Data are the means \pm SD of four mice. Statistically significant differences $(P<0 \cdot 05$ using Student's $t$ test) are indicated by: ${ }^{*}$, compared with negative control; ${ }^{* *}$, compared with pgD alone.

\begin{tabular}{|lccl|}
\hline Plasmid(s) & $\begin{array}{c}\text { Spontaneous } \\
\text { c.p.m. }\end{array}$ & $\begin{array}{c}\text { Experimental } \\
\text { c.p.m. }\end{array}$ & SI \\
\hline pcDNA3.1 & $5862 \pm 1354$ & $5287 \pm 1236$ & $0 \cdot 90 \pm 0 \cdot 28$ \\
pgD & $6518 \pm 1682$ & $22025 \pm 2357$ & $3 \cdot 38 \pm 0 \cdot 39^{\star}$ \\
pgD+pIL-18 & $7039 \pm 1768$ & $33869 \pm 4573$ & $4 \cdot 81 \pm 0 \cdot 67^{\star *}$ \\
\hline
\end{tabular}

\section{Results of viral challenge}

In control mice, epithelial lesion scores peaked on day 2 after viral challenge and then declined gradually. About 7 days after the challenge, all the epithelial lesions had recovered. However, pgD limited the lesion score to a level significantly lower than that observed in the negative control. When the mice were co-injected with pIL-18, the epithelial lesions were further reduced and the recovery time was also shortened significantly $(P<0 \cdot 05$; Fig. 2$)$.

\section{DISCUSSION}

Cytokines play important roles in the immune and inflammatory responses as indicators and regulators of the immune network (Cohen et al., 1998). Recombinant cytokines have been used clinically in the treatment of human diseases including cancers and infectious diseases (Nash et al., 1993; Opal et al., 1998; Bukowski, 2000). However, the short halflife of recombinant cytokines and the side effects due to

Table 1. ELISA detection of gD-specific $\lg G, \lg G 1$ and $\lg G 2 a$ isotypes after DNA immunization

Groups of mice $(n=4)$ were immunized with $100 \mu \mathrm{g}$ pgD alone or in combination with $100 \mu \mathrm{g}$ pIL18 at 0,2 and 4 weeks; pcDNA3.1-immunized mice were used as a negative control. Mice were bled 2 weeks after the final immunization and each serum was diluted $1: 200$ for isotype assay. Values for individual isotypes are $A_{490}$ (means $\left.\pm \mathrm{SD}\right)$. Statistically significant differences $(P<0.05$ using Student's $t$ test) are indicated by: ${ }^{*}$, compared with negative control; ${ }^{* *}$, compared with pgD alone.

\begin{tabular}{|lcccc|}
\hline Plasmid(s) & IgG & \multicolumn{1}{c}{ IgG1 } & \multicolumn{1}{c|}{ IgG2a } & IgG2a/IgG1 \\
\hline pcDNA3.1 & $0 \cdot 084 \pm 0 \cdot 005$ & $0 \cdot 056 \pm 0 \cdot 002$ & $0 \cdot 059 \pm 0 \cdot 004$ & $1 \cdot 05$ \\
pgD & $1 \cdot 348 \pm 0 \cdot 104^{\star}$ & $0 \cdot 222 \pm 0 \cdot 065^{\star}$ & $0 \cdot 897 \pm 0 \cdot 081^{\star}$ & $4 \cdot 04^{\star}$ \\
pgD+pIL-18 & $2 \cdot 976 \pm 0 \cdot 471^{\star *}$ & $0 \cdot 441 \pm 0 \cdot 140^{* *}$ & $2 \cdot 809 \pm 0 \cdot 458^{\star *}$ & $6 \cdot 37^{\star *}$ \\
\hline
\end{tabular}




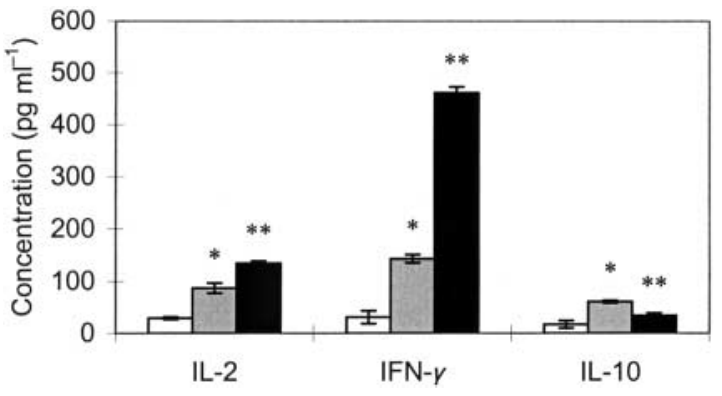

Fig. 1. Levels of cytokine production from splenocytes after gD stimulation in vitro. Groups of mice $(n=2)$ were immunized with $100 \mu \mathrm{g} \mathrm{pgD}$ alone (shaded bars) or in combination with $100 \mu \mathrm{g}$ plL18 (filled bars) at 0, 2 and 4 weeks; pcDNA3.1-immunized mice (open bars) were used as a negative control. Two weeks after the final immunization, mice were sacrificed and the splenocytes were isolated. Splenocytes were then stimulated with $10 \mu \mathrm{g} \mathrm{gD}$ protein $\mathrm{ml}^{-1}$ for 3 days. The experiment was repeated with similar results; data from the two experiments were included in the analysis. Data are the means $\pm S D$ of four mice. Statistically significant differences $(P<0.05$ using Student's $t$ test) are indicated by * (compared with negative control) or ${ }^{* *}$ (compared with pgD alone).

Table 3. Development of DTH in mice immunized with DNA vaccines

Groups of mice $(n=7)$ were immunized with $100 \mu \mathrm{g} p \mathrm{pg}$ alone or with $100 \mu \mathrm{g}$ pIL-18 at 0,2 and 4 weeks; pcDNA3.1-immunized mice were used as a negative control. Two weeks after the final immunization, mice were challenged with UV-inactivated HSV-1. Data are the means \pm SD. Statistically significant differences $(P<0.05$ using Student's $t$ test $)$ are indicated by: ${ }^{\star}$, compared with negative control; ${ }^{* *}$, compared with pgD alone.

\begin{tabular}{|lc|}
\hline Plasmid(s) & $\begin{array}{r}\text { Difference in thickness between left } \\
\text { and right pinnae }\left(\times 10^{\mathbf{2}} \mathbf{~ m m}\right)\end{array}$ \\
\hline pcDNA3.1 & $1 \cdot 00 \pm 2 \cdot 58$ \\
pgD & $11 \cdot 71 \pm 3 \cdot 44^{\star}$ \\
pgD + pIL-18 & $13 \cdot 14 \pm 3 \cdot 27^{\star *}$ \\
\hline
\end{tabular}

repetitive administration are still insoluble problems (Hara et al., 2000). Previous reports have shown that direct injection of cytokine genes into muscle resulted in the characteristic biological actions of these cytokines in vivo and could modulate immune responses (Iwasaki et al., 1997; Sin et al., 1999). However, there has been no study on the modulatory effect of using the human IL-18 gene as a molecular adjuvant to enhance the potency of DNA vaccine in an HSV-1 challenge model.

In the present study, we observed a significant increase in $\mathrm{gD}$ specific IgG production through vaccine modulation with an IL-18 expression plasmid. This is compatible with a previous report (Kim et al., 1998). Although both IgG2a and IgG1 were increased, the increase in IgG2a was more remarkable compared with that of IgG1 and resulted in a significantly

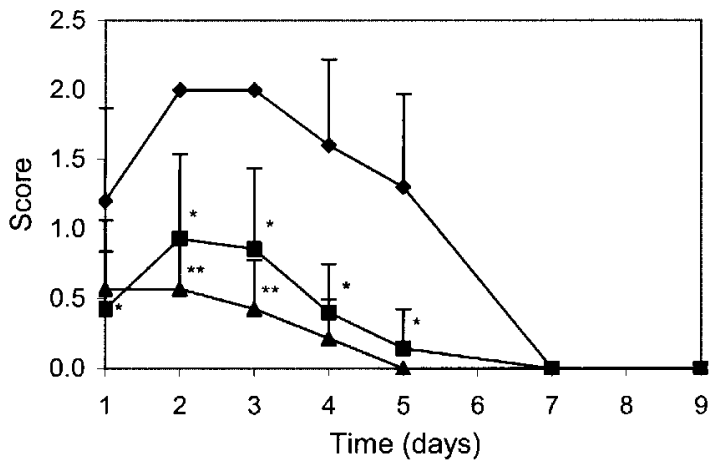

Fig. 2. Clinical scores for severity of epithelial keratitis. Groups of mice $(n=7)$ were immunized with $100 \mu \mathrm{g} \mathrm{pgD}$ alone $(\mathbf{a})$ or in combination with $100 \mu \mathrm{g} \mathrm{plL-18}(\mathbf{\Delta})$ at 0,2 and 4 weeks; pcDNA3.1-immunized mice $(\bullet)$ were used as a negative control. Two weeks after the final immunization, virus was instilled into the conjunctival sac as described in Methods. Values are mean scores and bars reflect SD. Statistically significant differences $(P<0.05$ using Kruskal-Wallis $\mathrm{H}$ test) are indicated by * (compared with negative control) or ** (compared with pgD alone).

increased IgG2a/IgG1 ratio. Since the IgG2a isotype is driven by Th1 cells, while the IgG1 isotype is driven by Th2 cells, this result suggested that $\mathrm{Th} 1 \mathrm{immune}$ responses were dominant when pIL-18 was co-injected. This was further verified by the cytokine production profile; we observed that co-injection with pIL-18 induced both IL- 2 and IFN- $\gamma$ secretion, but appeared to inhibit IL-10 production. Furthermore, significant increases of lymphocyte proliferation and the DTH response were achieved by co-injection of pIL-18. We also investigated the induction of a $\mathrm{gD}$-specific cytotoxic $\mathrm{T}$ lymphocyte (CTL) response by gD DNA vaccination, but no CTL activity was observed (data not shown), which was also consistent with previous reports (Ghiasi et al., 1995; Scott \& Trinchieri, 1997; Cruz et al., 1999). In our experiments, the adjuvant activity cannot be attributed to immunostimulatory sequences at the DNA level, as a preliminary experiment showed that mixing $\mathrm{pgD}$ with pcDNA3.1 vector did not demonstrate similar immunemodulatory function (data not shown). Thus, the use of an IL-18 expression plasmid in gD DNA vaccination may be an effective approach for inducing a serum antibody response as well as cell-mediated immune responses.

Previous reports have shown that humoral or cellular immune responses or both are responsible for protective immunity against HSV infection (Price et al., 1975; RagerZisman \& Allison, 1976; Nash \& Cambouropoulos, 1993). During primary infection, neutralizing antibodies can inactivate free virus particles (Notkins, 1974). On the other hand, HSV-specific antibodies, which are present at high levels in humans, are insufficient to prevent HSV latency in the central nervous system (McKendall, 1983). Furthermore, it has been suggested that HSV-specific cellular immunity, 
mediated particularly by $\mathrm{CD} 4^{+}$and not $\mathrm{CD} 8^{+}$cells, play a major role in eradicating HSV-infected cells and controlling recurrent HSV infection (Sethi et al., 1983; Sin et al., 1999).

In this study, co-injection of pIL-18 increased protection against HSV corneal challenge significantly, compared with pgD alone. By co-injecting pIL-18 with pgD, corneal lesion scores were decreased significantly and recovery from the herpetic lesion was speeded up. This protective immunity might be attributed to both humoral immunity, which is interpreted as increased production of gD-specific IgG, and cellular immunity, which is interpreted as increased splenocyte proliferation, DTH response and levels of cytokine (IL-2 and IFN- $\gamma$ ) production, when $\mathrm{pgD}$ was co-injected with pIL18. IFN- $\gamma$ might play a critical role in the protective immunity, since we observed that co-injection with pIL-18 induced significant IFN- $\gamma$ production from splenocytes in vitro. Many studies have revealed effects of IFN- $\gamma$ on HSV infection (Neumann-Haefelin et al., 1985; Geiger et al., 1995; Cantin et al., 1995). IFN- $\gamma$ takes effect in host resistance directly or via induction of an antiviral state in lymphocytes and macrophages (Landolfo et al., 1995). Furthermore, IFN$\gamma$ is also able to enhance NK cell activity, which has been reported to suppress HSV infection in vivo and in vitro (Reiter, 1993; Tanigawa et al., 2000).

The experiments described here demonstrate an enhanced immune response and protection against HSV challenge as measured 2 weeks after the final immunization. Clearly, it will be interesting to know how long these effects last in this animal model. This is the subject of further experimentation. Another issue that deserves to be addressed in future is whether this enhanced immune response might be preventive against establishment of HSV latency in the trigeminal ganglion, since this is the most distinct feature of this kind of virus.

In conclusion, the data presented here suggest that coinjection of an IL-18 expression plasmid with gD DNA vaccine could induce Th1-dominant immune responses efficiently, manifested as increases in the $\operatorname{IgG} 2 \mathrm{a} / \mathrm{IgG1}$ ratio, IL- 2 and IFN- $\gamma$ but a decrease in IL-10, and achieve better protection against HSV-1 challenge. This study also demonstrates the potential of the IL-18 gene as a molecular adjuvant, which appears promising for the prevention of infectious diseases.

\section{ACKNOWLEDGEMENTS}

This work was supported by grants from the Natural Science Foundation of Beijing and the Bureau of Health, Beijing, PR China.

\section{REFERENCES}

Billaut-Mulot, O., Idziorek, T., Loyens, M., Capron, A. \& Bahr, G. M. (2001). Modulation of cellular and humoral immune responses to a multiepitopic HIV-1 DNA vaccine by interleukin-18 DNA immunization/viral protein boost. Vaccine 19, 2803-2811.

Bourne, N., Stanberry, L. R., Bernstein, D. I. \& Lew, D. (1996). DNA immunization against experimental genital herpes simplex virus infection. J Infect Dis 173, 800-807.
Bukowski, R. M. (2000). Cytokine combinations: therapeutic use in patients with advanced renal cell carcinoma. Semin Oncol 27, 204-212.

Cantin, E. M., Hinton, D. R., Chen, J. \& Openshaw, H. (1995). Gamma interferon expression during acute and latent nervous system infection by herpes simplex virus type 1. J Virol 69, 4898-4905.

Cohen, A. D., Boyer, J. D. \& Weiner, D. B. (1998). Modulating the immune response to genetic immunization. FASEB J 12, 1611-1626.

Corey, L., Langenberg, A. G., Ashley, R. \& 14 other authors (1999). Recombinant glycoprotein vaccine for the prevention of genital HSV-2 infection: two randomized controlled trials. Chiron HSV Vaccine Study Group. JAMA 282, 331-340.

Cruz, P. E., Khalil, P. L., Dryden, T. D., Chiou, H. C., Fink, P. S., Berberich, S. J. \& Bigley, N. J. (1999). A novel immunization method to induce cytotoxic T-lymphocyte responses (CTL) against plasmid-encoded herpes simplex virus type-1 glycoprotein D. Vaccine 17, 1091-1099.

Fujioka, N., Akazawa, R., Ohashi, K., Fujii, M., Ikeda, M. \& Kurimoto, M. (1999). Interleukin-18 protects mice against acute herpes simplex virus type 1 infection. J Virol 73, 2401-2409.

Garcia, V. E., Uyemura, K., Sieling, P. A., Ochoa, M. T., Morita, C. T., Okamura, H., Kurimoto, M., Rea, T. H. \& Modlin, R. L. (1999). IL-18 promotes type 1 cytokine production from NK cells and $\mathrm{T}$ cells in human intracellular infection. J Immunol 162, 6114-6121.

Geiger, K. D., Lee, M. S., Baugh, C. \& Sarvetnick, N. E. (1995). Protective effects of interferon- $\gamma$ in intraocular herpes simplex type 1 infection do not depend on major histocompatibility complex class I or class II expression. J Neurovirol 1, 405-409.

Ghiasi, H., Cai, S., Slanina, S., Nesburn, A. B. \& Wechsler, S. L. (1995). Vaccination of mice with herpes simplex virus type 1 glycoprotein $D$ DNA produces low levels of protection against lethal HSV-1 challenge. Antiviral Res 28, 147-157.

Hara, I., Nagai, H., Miyake, H. \& 8 other authors (2000). Effectiveness of cancer vaccine therapy using cells transduced with the interleukin-12 gene combined with systemic interleukin-18 administration. Cancer Gene Ther 7, 83-90.

Inoue, T., Inoue, Y., Nakamura, T. \& 8 other authors (2000). Preventive effect of local plasmid DNA vaccine encoding gD or gD-IL-2 on herpetic keratitis. Invest Ophthalmol Vis Sci 41, 4209-4215.

Iwasaki, A., Stiernholm, B. J., Chan, A. K., Berinstein, N. L. \& Barber, B. H. (1997). Enhanced CTL responses mediated by plasmid DNA immunogens encoding costimulatory molecules and cytokines. J Immunol 158, 4591-4601.

Keadle, T. L., Laycock, K. A., Miller, J. K., Hook, K. K., Fenoglio, E. D., Francotte, M., Slaoui, M., Stuart, P. M. \& Pepose, J. S. (1997). Efficacy of a recombinant glycoprotein $\mathrm{D}$ subunit vaccine on the development of primary and recurrent ocular infection with herpes simplex virus type 1 in mice. J Infect Dis 176, 331-338.

Kim, J. J., Bagarazzi, M. L., Trivedi, N. \& 12 other authors (1997). Engineering of in vivo immune responses to DNA immunization via codelivery of costimulatory molecule genes. Nat Biotechnol 15, 641-646.

Kim, J. J., Trivedi, N. N., Nottingham, L. K. \& 13 other authors (1998). Modulation of amplitude and direction of in vivo immune responses by co-administration of cytokine gene expression cassettes with DNA immunogens. Eur J Immunol 28, 1089-1103.

Kim, J. J., Simbiri, K. A., Sin, J. I. \& 10 other authors (1999a). Cytokine molecular adjuvants modulate immune responses induced by DNA vaccine constructs for HIV-1 and SIV. J Interferon Cytokine Res 19, 77-84.

Kim, J. J., Nottingham, L. K., Tsai, A. \& 9 other authors (1999b). Antigen-specific humoral and cellular immune responses can be modulated in rhesus macaques through the use of IFN- $\gamma$, IL-12, or IL-18 gene adjuvants. J Med Primatol 28, 214-223. 
Kim, J. J., Yang, J. S., Dentchev, T., Dang, K. \& Weiner, D. B. (2000), Chemokine gene adjuvants can modulate immune responses induced by DNA vaccines. J Interferon Cytokine Res 20, 487-498.

Kohno, K., Kataoka, J., Ohtsuki, T., Suemoto, Y., Okamoto, I., Usui, M., Ikeda, M. \& Kurimoto, M. (1997). IFN- $\gamma$-inducing factor (IGIF) is a costimulatory factor on the activation of Th1 but not Th2 cells and exerts its effect independently of IL-12. J Immunol 158, 1541-1550.

Kremer, L., Dupre, L., Wolowczuk, I. \& Locht, C. (1999). In vivo immunomodulation following intradermal injection with DNA encoding IL-18. J Immunol 163, 3226-3231.

Landolfo, S., Gribaudo, G., Angeretti, A. \& Gariglio, M. (1995). Mechanisms of viral inhibition by interferons. Pharmacol Ther $\mathbf{6 5}$, 415-442.

McKendall, R. R. (1983). Delayed IgG-mediated clearance of herpes simplex virus type 1 from the CNS but not footpad during the early stages of infection: possible result of relative integrity of the bloodbrain barrier. J Gen Virol 64, 1965-1972.

Nash, A. A. \& Cambouropoulos, P. (1993). The immune response to herpes simplex virus. Semin Virol 4, 181-186.

Nash, A. D., Lofthouse, S. A., Barcham, G. J., Jacobs, H. J., Ashman, K., Meeusen, E. N., Brandon, M. R. \& Andrews, A. E. (1993). Recombinant cytokines as immunological adjuvants. Immunol Cell Biol 71, 367-379.

Neumann-Haefelin, D., Sundmacher, R., Frey, H. \& Merk, W. (1985). Recombinant HuIFN- $\gamma$ prevents herpes simplex keratitis in African green monkeys: demonstration of synergism with recombinant HuIFN$\alpha$ 2. Med Microbiol Immunol (Berl) 174, 81-86.

Notkins, A. L. (1974). Immune mechanisms by which the spread of viral infections is stopped. Cell Immunol 11, 478-483.

Ohkusu, K., Yoshimoto, T., Takeda, K., Ogura, T., Kashiwamura, S., Iwakura, Y., Akira, S., Okamura, H. \& Nakanishi, K. (2000). Potentiality of interleukin-18 as a useful reagent for treatment and prevention of Leishmania major infection. Infect Immun 68, 2449-2456.

Okamura, H., Nagata, K., Komatsu, T. \& 8 other authors (1995). A novel costimulatory factor for gamma interferon induction found in the livers of mice causes endotoxic shock. Infect Immun 63, 3966-3972.

Opal, S. M., Wherry, J. C. \& Grint, P. (1998). Interleukin-10: potential benefits and possible risks in clinical infectious diseases. Clin Infect Dis 27, 1497-1507.
Price, R. W., Walz, M. A., Wohlenberg, C. \& Notkins, A. L. (1975). Latent infection of sensory ganglia with herpes simplex virus: efficacy of immunization. Science 188, 938-940.

Rager-Zisman, B. \& Allison, A. C. (1976). Mechanism of immunologic resistance to herpes simplex virus 1 (HSV-1) infection. J Immunol 116, $35-40$.

Reiter, Z. (1993). Interferon - a major regulator of natural killer cellmediated cytotoxicity. J Interferon Res 13, 247-257.

Scott, P. \& Trinchieri, G. (1997). IL-12 as an adjuvant for cell-mediated immunity. Semin Immunol 9, 285-291.

Sethi, K. K., Omata, Y. \& Schneweis, K. E. (1983). Protection of mice from fatal herpes simplex virus type 1 infection by adoptive transfer of cloned virus-specific and H-2-restricted cytotoxic T lymphocytes. J Gen Virol 64, 443-447.

Sin, J. I., Kim, J. J., Ugen, K. E., Ciccarelli, R. B., Higgins, T. J. \& Weiner, D. B. (1998). Enhancement of protective humoral (Th2) and cell-mediated (Th1) immune responses against herpes simplex virus- 2 through co-delivery of granulocyte-macrophage colony-stimulating factor expression cassettes. Eur J Immunol 28, 3530-3540.

Sin, J. I., Kim, J. J., Arnold, R. L. \& 9 other authors (1999). IL-12 gene as a DNA vaccine adjuvant in a herpes mouse model: IL-12 enhances Th1type $\mathrm{CD} 4{ }^{+} \mathrm{T}$ cell-mediated protective immunity against herpes simplex virus-2 challenge. J Immunol 162, 2912-2921.

Tanigawa, M., Bigger, J. E., Kanter, M. Y. \& Atherton, S. S. (2000). Natural killer cells prevent direct anterior-to-posterior spread of herpes simplex virus type 1 in the eye. Invest Ophthalmol Vis Sci 41, 132-137.

Tovey, M. G., Meritet, J. F., Guymarho, J. \& Maury, C. (1999). Mucosal cytokine therapy: marked antiviral and antitumor activity. J Interferon Cytokine Res 19, 911-921.

Ushio, S., Namba, M., Okura, T. \& 13 other authors (1996). Cloning of the cDNA for human IFN- $\gamma$-inducing factor, expression in Escherichia coli, and studies on the biologic activities of the protein. J Immunol 156, 4274-4279.

Zhu, M. Z., Liu, H. W., Liu, X. J., Han, Y. H., Yang, B. L. \& Song, G. X. (2002). Construction of herpes simplex virus type 1 glycoprotein $D$ DNA vaccine and its preliminary study. Acta Acad Med Sin 24, 67-70. 